\title{
A new technique for convergence theorem of fixed point problem of quasi-nonexpansive mapping
}

Kanyarat Cheawchan ${ }^{1}$, Suthep Suantai ${ }^{2}$ and Atid Kangtunyakarn ${ }^{\text {* }}$

\section{"Correspondence:}

beawrock@hotmail.com

'Department of Mathematics,

Faculty of Science, King Mongkut's

Institute of Technology Ladkrabang,

Bangkok 10520, Thailand

Full list of author information is

available at the end of the article

\begin{abstract}
For the purpose of this paper, we use the method different from the relaxed extragradient method for finding a common element of the set of fixed points of a quasi-nonexpansive mapping, the set of solutions of equilibrium problems, and the set of solutions of a modified system of variational inequalities without demiclosed condition of $W$ and $W_{\omega}:=(1-\omega) l+\omega W$, where $W$ is a quasi-nonexpansive mapping and $\omega \in\left(0, \frac{1}{2}\right)$ in the framework of Hilbert space. By using our main result, we obtain a strong convergence theorem involving a finite family of nonspreading mappings and another corollary. Moreover, we give a numerical example to encourage our main theorem.
\end{abstract}

Keywords: quasi-nonexpansive mapping; equilibrium problem; variational inequality problem; fixed point problem

\section{Introduction}

Let $C$ be a nonempty closed convex subset of a real Hilbert space $H$. Recall that the mapping $W: C \rightarrow C$ is called quasi-nonexpansive if

$$
\|W p-q\| \leq\|p-q\|
$$

for all $p \in C$ and $q \in F(W)$. We denote by $F(W)$ the set of fixed points of $W$. Fixed point problems have been widely studied and developed in the literature.

Let $\Psi$ be a bifunction of $C \times C$ into $\mathbb{R}$, where $\mathbb{R}$ is the set of real numbers. The equilibrium problem for $\Psi: C \times C \rightarrow \mathbb{R}$ is to find $p \in C$ such that

$$
\Psi(p, \zeta) \geq 0, \quad \forall \zeta \in C
$$

We denote the set of solutions of (1.1) by $\mathrm{EP}(\Psi)$. Equilibrium problems were introduced by Blum and Oettli [1] in 1994 and included many well-known problems such as the variational inequality problem, the optimization problem, and the nonexpansive mapping and fixed point problem.

(c) 2015 Cheawchan et al. This article is distributed under the terms of the Creative Commons Attribution 4.0 International License (http://creativecommons.org/licenses/by/4.0/), which permits unrestricted use, distribution, and reproduction in any medium, provided you give appropriate credit to the original author(s) and the source, provide a link to the Creative Commons license, and indicate if changes were made. 
A mapping $D_{1}: C \rightarrow H$ is called $d_{1}$-inverse strongly monotone if there exists a positive real number $d_{1}>0$ such that

$$
\left\langle D_{1} p-D_{1} \zeta, p-\zeta\right\rangle \geq d_{1}\left\|D_{1} p-D_{1} \zeta\right\|^{2}
$$

for all $p, \zeta \in C$.

Let $B: C \rightarrow H$. The variational inequality is to find a point $\phi \in C$ such that

$$
\langle B \phi, \psi-\phi\rangle \geq 0,
$$

for all $\psi \in C$. The set of solutions of (1.2) is denoted by $\operatorname{VIP}(C, B)$. The variational inequalities were initially studied and introduced by Lions and Stampacchia [2].

The concept of quasi-nonexpansive mapping was investigated by Diaz and Metcalf [3]. In 2007, Su et al. [4] introduced strong convergence theorems for quasi-nonexpansive mappings, the monotone hybrid iteration method used to approximate the fixed point of quasi-nonexpansive mappings. In 2011, Tian and Jin [5] introduced an iterative method of a quasi-nonexpansive mapping in the framework of Hilbert space. They proved the strong convergence theorem of iterative scheme $\left\{p_{n}\right\}$ generated by (1.3) as follows.

Theorem 1.1 Let $H$ be a real Hilbert space, let $F$ be a $\kappa$-Lipschitzian and $\eta$-strongly monotone operator on $H$ with $\kappa>0, \eta>0$ and let $W$ be a quasi-nonexpansive mapping on $H$, and $f$ is a L-Lipschitzian mapping with coefficient $L>0$ for all $p, \zeta \in H$. Assume the set $F(W)$ of fixed points of $W$ is nonempty closed and convex. Let $0<\mu<\frac{2 \eta}{\kappa^{2}}, 0<\gamma<\mu\left(\eta-\frac{\mu \kappa^{2}}{2}\right) / L=\tau / L$ and start with an arbitrary chosen $p_{0} \in H$, let the sequence $\left\{p_{n}\right\}$ be generated by

$$
p_{n+1}=\alpha_{n} \gamma f\left(p_{n}\right)+\left(I-\alpha_{n} \mu F\right) W_{\omega} p_{n}
$$

where the sequence $\left\{\alpha_{n}\right\} \subset(0,1)$ satisfies $\lim _{n \rightarrow \infty} \alpha_{n}=0$, and $\sum_{n=1}^{\infty} \alpha_{n}=\infty$. Also $\omega \in\left(0, \frac{1}{2}\right)$, $W_{\omega}:=(1-\omega) I+\omega W$ with two conditions on $W$ :

1. $\|W p-q\| \leq\|p-q\|$ for any $p \in H$, and $q \in F(W)$; this means that $W$ is a quasi-nonexpansive mapping;

2. $W$ is demiclosed on $H$; that is, if $\left\{\zeta_{k}\right\} \subset H, \zeta_{k} \rightarrow \xi$, and $(I-W) \zeta_{k} \rightarrow 0$, then $\xi \in F(W)$. Then $\left\{p_{n}\right\}$ converges strongly to the $p^{*} \in F(W)$ which is the unique solution of the VIP:

$$
\left\langle(\mu F-\gamma f) p^{*}, p-p^{*}\right\rangle \leq 0, \quad \forall p \in F(W) .
$$

Many strong convergence theorems of quasi-nonexpansive mapping $W$ were proved by assuming the following conditions:

1. $W_{\omega}:=(1-\omega) I+\omega W$ for all $\omega \in\left(0, \frac{1}{2}\right)$,

2. $W$ is demiclosed on $H$.

In 2012, Dong et al. [6] proved strong convergence theorem by using a relaxed extragradient method as follows.

Theorem 1.2 Let $C$ be a nonempty closed convex subset of a real Hilbert space H. Let the mappings $D_{1}, D_{2}: C \rightarrow H$ be $d_{1}$-inverse strongly monotone and $d_{2}$-inverse strongly monotone, respectively. Let $\Psi$ be a bifunction from $C \times C \rightarrow \mathbb{R}$ satisfying (J1)-(J4) and let $\left\{W_{n}\right\}_{n=1}^{\infty}: C \rightarrow C$ be a countable family of nonexpansive mappings such that $\Omega:=$ 
$\bigcap_{n=1}^{\infty} F\left(W_{n}\right) \cap \operatorname{EP}(\Psi) \cap F(G) \neq \emptyset$. Let $f: C \rightarrow C$ be a contraction with coefficient $\rho \in(0,1 / 2)$. Set $\beta_{0}=1$. For given $p_{1} \in C$ arbitrarily, let the sequences $\left\{p_{n}\right\},\left\{\zeta_{n}\right\},\left\{\xi_{n}\right\}$, and $\left\{\phi_{n}\right\}$ be generated by

$$
\left\{\begin{array}{l}
\Psi\left(\phi_{n}, \zeta\right)+\frac{1}{g_{n}}\left\langle\zeta-\phi_{n}, \phi_{n}-p_{n}\right\rangle \geq 0, \quad \forall \zeta \in C \\
\xi_{n}=P_{C}\left(\phi_{n}-\lambda_{B} D_{2} \phi_{n}\right) \\
\zeta_{n}=\alpha_{n} f\left(p_{n}\right)+\left(1-\alpha_{n}\right) P_{C}\left(\xi_{n}-\lambda_{A} D_{1} \xi_{n}\right), \\
p_{n+1}=\beta_{n} p_{n}+\sigma_{n} \sum_{i=1}^{\infty}\left(\beta_{i-1}-\beta_{i}\right) W_{i} \zeta_{n} \\
\quad+\left(1-\beta_{n}\right)\left(1-\sigma_{n}\right) P_{C}\left(\xi_{n}-\lambda_{A} D_{1} \xi_{n}\right), \quad \forall n \in \mathbb{N}
\end{array}\right.
$$

where $\lambda_{A} \in\left(0,2 d_{1}\right), \lambda_{B} \in\left(0,2 d_{2}\right)$, and the sequences $\left\{\alpha_{n}\right\} \subset[0,1],\left\{\beta_{n}\right\} \subset[0,1],\left\{\sigma_{n}\right\} \subset[0,1]$, and $\left\{g_{n}\right\} \subset(r, \infty), r>0$, are such that

(i) $\left\{\beta_{n}\right\}$ is strictly decreasing,

(ii) $0<\liminf _{n \rightarrow \infty} \beta_{n}<\limsup _{n \rightarrow \infty} \beta_{n}<1$,

(iii) $\lim _{n \rightarrow \infty} \alpha_{n}=0$ and $\sum_{n=1}^{\infty} \alpha_{n}=\infty$,

(iv) $\sigma_{n}>1 / 2(1-\rho), \sum_{n=1}^{\infty}\left|\sigma_{n}-\sigma_{n-1}\right|<\infty$,

(v) $\sum_{n=1}^{\infty}\left|g_{n}-g_{n-1}\right|<\infty$.

Then the sequence $\left\{p_{n}\right\}$ generated by (1.4) converges strongly to $p^{*}=P_{\Omega} \cdot f\left(p^{*}\right)$, and $\left(p^{*}, \zeta^{*}\right)$ is a solution of the general system of variational inequalities $(1.5)$ where $\zeta^{*}=P_{C}\left(p^{*}-\lambda_{B} D_{2} p^{*}\right)$.

Many authors used the extragradient method to prove fixed point theorem of nonlinear mappings.

Let $D_{1}, D_{2}: C \rightarrow H$ be two mappings. In 2008, Ceng et al. [7] introduced a relaxed extragradient method for finding solutions of problem $\left(p^{*}, \xi^{*}\right) \in C \times C$ such that

$$
\begin{cases}\left\langle\lambda_{A} D_{1} \xi^{*}+p^{*}-\xi^{*}, p-p^{*}\right\rangle \geq 0, & \forall p \in C, \\ \left\langle\lambda_{B} D_{2} p^{*}+\xi^{*}-p^{*}, p-\xi^{*}\right\rangle \geq 0, & \forall p \in C,\end{cases}
$$

which is called a system of variational inequalities where $\lambda_{A}, \lambda_{B}>0$.

In 2013, Kangtunyakarn [8] modified (1.5) for finding $\left(p^{*}, \xi^{*}\right) \in C \times C$ such that

$$
\left\{\begin{array}{l}
\left\langle p^{*}-\left(I-\lambda_{A} D_{1}\right)\left(a p^{*}+(1-a) \xi^{*}\right), p-p^{*}\right\rangle \geq 0, \quad \forall p \in C, \\
\left\langle\xi^{*}-\left(I-\lambda_{B} D_{2}\right) p^{*}, p-\xi^{*}\right\rangle \geq 0, \quad \forall p \in C
\end{array}\right.
$$

which is called a modification of system of variational inequalities, for every $\lambda_{A}, \lambda_{B}>0$ and $a \in[0,1]$. If $a=0,(1.6)$ reduces to (1.5). He introduced the relation between solutions of (1.6) and fixed point of the mapping $G$ as follows.

Lemma 1.3 Let $C$ be a nonempty closed convex subset of a real Hilbert space $H$ and let $D_{1}, D_{2}: C \rightarrow H$ be mappings. For every $\lambda_{A}, \lambda_{B}>0$ and $a \in[0,1]$, the following statements are equivalent:

1. $\left(p^{*}, \xi^{*}\right) \in C \times C$ is a solution of problem (1.6),

2. $p^{*}$ is a fixed point of the mapping $G: C \rightarrow C$, i.e., $p^{*} \in F(G)$, defined by

$$
G(p)=P_{C}\left(I-\lambda_{A} D_{1}\right)\left(a p+(1-a) P_{C}\left(I-\lambda_{B} D_{2}\right) p\right)
$$

where $\xi^{*}=P_{C}\left(I-\lambda_{B} D_{2}\right) p^{*}$. 
After we investigated Theorem 1.1, Theorem 1.2 and researchers in the same direction, we have the questions as follows:

(1) Can we prove strong convergence theorem without demiclosed condition and $W_{\omega}:=(1-\omega) I+\omega W$, where $W$ is a quasi-nonexpansive mapping and $\omega \in\left(0, \frac{1}{2}\right)$ in the framework of Hilbert space?

(2) Can we prove strong convergence theorem without relaxed extragradient method? In this paper, we give the answer for the mentioned questions and introduce the method of iterative scheme $\left\{p_{n}\right\}$ for finding a common element of the set of fixed points of a quasinonexpansive mapping, the set of solutions of equilibrium problems and the set of solutions of a modified system of variational inequalities. Applying our main result, we prove strong convergence theorem involving a finite family of nonspreading mappings and another corollary. Moreover, We also give a numerical example to support our main theorem.

\section{Preliminaries}

Let $H$ be a real Hilbert space with inner product $\langle\cdot, \cdot\rangle$ and norm $\|\cdot\|$. In this paper, we use the symbol of weak and strong convergence by ' $\rightarrow$ ' and ' $\rightarrow$ ', respectively. For every $p \in H$, there exists a unique nearest point $P_{C} p$ in $C$ such that $\left\|p-P_{C} p\right\| \leq\|p-\zeta\|$ for all $\zeta \in C$. $P_{C}$ is called the metric projection of $H$ onto $C$.

Remark 2.1 It is well known that metric projection $P_{C}$ has the following properties:

1. $P_{C}$ is firmly nonexpansive, i.e.,

$$
\left\|P_{C} p-P_{C} \zeta\right\|^{2} \leq\left\langle P_{C} p-P_{C} \zeta, p-\zeta\right\rangle, \quad \forall p, \zeta \in H
$$

2. For each $p \in H$,

$$
\xi=P_{C}(p) \quad \Leftrightarrow \quad\langle p-\xi, \xi-\zeta\rangle \geq 0, \quad \forall \zeta \in C
$$

Recall that $H$ satisfies Opial's condition [9], i.e., for any sequence $\left\{p_{n}\right\}$ with $p_{n} \rightarrow p$, the inequality

$$
\lim _{n \rightarrow \infty} \inf \left\|p_{n}-p\right\|<\lim _{n \rightarrow \infty} \inf \left\|p_{n}-\zeta\right\|
$$

holds for every $\zeta \in H$ with $\zeta \neq p$.

Lemma 2.2 Let $H$ be a real Hilbert space. Then we have the following well-known results:

1. $\|p \pm \zeta\|^{2}=\|p\|^{2} \pm 2\langle p, \zeta\rangle+\|\zeta\|^{2}$,

2. $\|p+\zeta\|^{2} \leq\|p\|^{2}+2\langle\zeta, p+\zeta\rangle$,

for all $p, \zeta \in H$.

Lemma 2.3 ([10]) Let $(E,\langle\cdot, \cdot\rangle)$ be an inner product space. Then, for all $p, \zeta, \xi \in E$ and $\alpha_{1}, \alpha_{2}, \alpha_{3} \in[0,1]$ with $\alpha_{1}+\alpha_{2}+\alpha_{3}=1$, we have

$$
\begin{aligned}
\left\|\alpha_{1} p+\alpha_{2} \zeta+\alpha_{3} \xi\right\|^{2}= & \alpha_{1}\|p\|^{2}+\alpha_{2}\|\zeta\|^{2}+\alpha_{3}\|\xi\|^{2}-\alpha_{1} \alpha_{2}\|p-\zeta\|^{2} \\
& -\alpha_{1} \alpha_{3}\|p-\xi\|^{2}-\alpha_{2} \alpha_{3}\|\zeta-\xi\|^{2}
\end{aligned}
$$


For solving the equilibrium problem, we assume that the bifunction $\Psi: C \times C \rightarrow \mathbb{R}$ satisfies the following conditions:

(J1) $\Psi(p, p)=0$ for all $p \in C$;

(J2) $\Psi$ is monotone, i.e., $\Psi(p, \zeta)+\Psi(\zeta, p) \leq 0$ for all $p, \zeta \in C$;

(J3) for each $p, \zeta, \xi \in C$,

$$
\lim _{t \downarrow 0} \Psi(t \xi+(1-t) p, \zeta) \leq \Psi(p, \zeta)
$$

(J4) for each $p \in C, \zeta \mapsto \Psi(p, \zeta)$ is convex and lower semicontinuous.

Lemma 2.4 ([1]) Let $C$ be a nonempty closed convex subset of $H$ and let $\Psi$ be a bifunction of $C \times C$ into $\mathbb{R}$ satisfying (J1)-(J4). Let $r>0$ and $p \in H$. Then there exists $\xi \in C$ such that

$$
\Psi(\xi, \zeta)+\frac{1}{r}\langle\zeta-\xi, \xi-p\rangle \geq 0, \quad \forall \zeta \in C
$$

Lemma 2.5 ([11]) Assume that $\Psi: C \times C \rightarrow \mathbb{R}$ satisfies (J1)-(J4). For $r>0$, define a mapping $W_{r}: H \rightarrow C$ as follows:

$$
W_{r}(p)=\left\{\xi \in C: \Psi(\xi, \zeta)+\frac{1}{r}\langle\zeta-\xi, \xi-p\rangle \geq 0, \forall \zeta \in C\right\}
$$

for all $p \in H$. Then the following hold:

(1) $W_{r}$ is single-valued;

(2) $W_{r}$ is firmly nonexpansive, i.e., for any $p, \zeta \in H$,

$$
\left\|W_{r}(p)-W_{r}(\zeta)\right\|^{2} \leq\left\langle W_{r}(p)-W_{r}(\zeta), p-\zeta\right\rangle
$$

(3) $F\left(W_{r}\right)=\mathrm{EP}(\Psi)$;

(4) $\mathrm{EP}(\Psi)$ is closed and convex.

Lemma 2.6 ([12]) Let $\left\{h_{n}\right\}$ be a sequence of nonnegative real numbers satisfying

$$
h_{n+1} \leq\left(1-\alpha_{n}\right) h_{n}+\delta_{n}, \quad \forall n \geq 0,
$$

where $\left\{\alpha_{n}\right\}$ is a sequence in $(0,1)$ and $\left\{\delta_{n}\right\}$ is a sequence such that

(1) $\sum_{n=1}^{\infty} \alpha_{n}=\infty$,

(2) $\lim \sup _{n \rightarrow \infty} \frac{\delta_{n}}{\alpha_{n}} \leq 0$ or $\sum_{n=1}^{\infty}\left|\delta_{n}\right|<\infty$.

Then $\lim _{n \rightarrow \infty} h_{n}=0$.

Lemma 2.7 ([13]) Let $H$ be a real Hilbert space, let $C$ be a nonempty closed convex subset of $H$ and let $D_{1}$ be a mapping of $C$ into $H$. Let $u \in C$. Then for $\lambda>0$,

$$
u=P_{C}\left(I-\lambda D_{1}\right) u \quad \Leftrightarrow \quad u \in \operatorname{VIP}\left(C, D_{1}\right),
$$

where $P_{C}$ is the metric projection of $H$ onto $C$. 
Lemma 2.8 ([14]) Let $C$ be a nonempty closed convex subset of a real Hilbert space $H$ and let $W: C \rightarrow C$ be a quasi-nonexpansive mapping with $F(W) \neq \emptyset$. Then $\operatorname{VIP}(C, I-W)=$ $F(W)$.

Remark 2.9 From Lemmas 2.7 and 2.8, we have

$$
F(W)=\operatorname{VIP}(C, I-W)=F\left(P_{C}(I-\lambda(I-W))\right)
$$

for all $\lambda>0$.

\section{Main result}

Theorem 3.1 Let $C$ be a nonempty closed convex subset of a real Hilbert space $H$, let $\Psi_{1}, \Psi_{2}: C \times C \rightarrow \mathbb{R}$ be bifunctions satisfying (J1)-(J4) and let $W: C \rightarrow C$ be a quasinonexpansive mapping. Let $D_{1}, D_{2}: C \rightarrow H$ be $d_{1}, d_{2}$-inverse strongly monotone mappings, respectively. Define the mapping $G: C \rightarrow C$ by $G(p)=P_{C}\left(I-\lambda_{A} D_{1}\right)\left(a p+(1-a) P_{C}(I-\right.$ $\left.\left.\lambda_{B} D_{2}\right) p\right)$ for all $p \in C$ and $a \in[0,1]$. Assume $\mathcal{F}=\mathrm{EP}\left(\Psi_{1}\right) \cap \operatorname{EP}\left(\Psi_{2}\right) \cap F(G) \cap F(W) \neq \emptyset$. Suppose that $p_{1}, u \in C$ and let $\left\{p_{n}\right\},\left\{\phi_{n}\right\}$, and $\left\{\psi_{n}\right\}$ be sequences generated by

$$
\left\{\begin{array}{l}
\Psi_{1}\left(\phi_{n}, \zeta\right)+\frac{1}{g_{n}}\left\langle\zeta-\phi_{n}, \phi_{n}-p_{n}\right\rangle \geq 0, \quad \forall \zeta \in C, \\
\Psi_{2}\left(\psi_{n}, \zeta\right)+\frac{1}{h_{n}}\left\langle\zeta-\psi_{n}, \psi_{n}-p_{n}\right\rangle \geq 0, \quad \forall \zeta \in C, \\
p_{n+1}=\alpha_{n} u+\beta_{n} p_{n}+\gamma_{n} P_{C}\left(I-\lambda_{n}(I-W)\right) \phi_{n}+\delta_{n} G\left(\psi_{n}\right), \quad \forall n \in \mathbb{N},
\end{array}\right.
$$

where the sequences $\lambda_{A} \in\left(0,2 d_{1}\right), \lambda_{B} \in\left(0,2 d_{2}\right)$ and $\left\{\alpha_{n}\right\},\left\{\beta_{n}\right\},\left\{\gamma_{n}\right\},\left\{\delta_{n}\right\} \subseteq[0,1]$ with $\alpha_{n}+$ $\beta_{n}+\gamma_{n}+\delta_{n}=1$ for all $n \in \mathbb{N}$. Suppose the following conditions hold:

(i) $\lim _{n \rightarrow \infty} \alpha_{n}=0$ and $\sum_{n=1}^{\infty} \alpha_{n}=\infty$,

(ii) $0<c \leq \beta_{n}, \gamma_{n}, \delta_{n} \leq d<1$ for some $c, d>0$ and for all $n \geq 1$,

(iii) $0<e \leq g_{n}, h_{n} \leq f$ for some $e, f>0$ and for all $n \geq 1$,

(iv) $\sum_{n=1}^{\infty} \lambda_{n}<\infty$ and $0<\lambda_{n}<1$,

(v) $\sum_{n=1}^{\infty}\left|\alpha_{n+1}-\alpha_{n}\right|<\infty, \sum_{n=1}^{\infty}\left|\beta_{n+1}-\beta_{n}\right|<\infty, \sum_{n=1}^{\infty}\left|\gamma_{n+1}-\gamma_{n}\right|<\infty$, $\sum_{n=1}^{\infty}\left|\lambda_{n+1}-\lambda_{n}\right|<\infty, \sum_{n=1}^{\infty}\left|g_{n+1}-g_{n}\right|<\infty, \sum_{n=1}^{\infty}\left|h_{n+1}-h_{n}\right|<\infty$.

Then $\left\{p_{n}\right\},\left\{\phi_{n}\right\}$, and $\left\{\psi_{n}\right\}$ converge strongly to $p_{0}=P_{\mathcal{F}} u$ and $\left(p_{0}, \xi_{0}\right)$ is a solution of (1.6) where $\xi_{0}=P_{C}\left(I-\lambda_{B} D_{2}\right) p_{0}$.

Proof First, we show that $G$ is a nonexpansive mapping. Let $p, \zeta \in C$. Since $D_{1}, D_{2}$ are $d_{1}, d_{2}$-inverse strongly monotone, $\lambda_{A} \in\left(0,2 d_{1}\right)$, and $\lambda_{B} \in\left(0,2 d_{2}\right)$, we have

$$
\begin{aligned}
\| & \left(I-\lambda_{A} D_{1}\right) p-\left(I-\lambda_{A} D_{1}\right) \zeta \|^{2} \\
& =\|p-\zeta\|^{2}-2 \lambda_{A}\left\langle p-\zeta, D_{1} p-D_{1} \zeta\right\rangle+\lambda_{A}^{2}\left\|D_{1} p-D_{1} \zeta\right\|^{2} \\
& \leq\|p-\zeta\|^{2}-2 d_{1} \lambda_{A}\left\|D_{1} p-D_{1} \zeta\right\|^{2}+\lambda_{A}^{2}\left\|D_{1} p-D_{1} \zeta\right\|^{2} \\
& =\|p-\zeta\|^{2}+\lambda_{A}\left(\lambda_{A}-2 d_{1}\right)\left\|D_{1} p-D_{1} \zeta\right\|^{2} \\
& \leq\|p-\zeta\|^{2} .
\end{aligned}
$$

Then $I-\lambda_{A} D_{1}$ is a nonexpansive mapping. Similarly $I-\lambda_{B} D_{2}$ is a nonexpansive mapping. Then $G$ is a nonexpansive mapping. 
Next, we show $\left\{p_{n}\right\}$ is bounded. Let $\xi \in \mathcal{F}$, then $\phi_{n}=W_{g_{n}} p_{n}$ and $\psi_{n}=W_{h_{n}} p_{n}$. It is clear that $\left\|\phi_{n}-\xi\right\| \leq\left\|p_{n}-\xi\right\|$ and $\left\|\psi_{n}-\xi\right\| \leq\left\|p_{n}-\xi\right\|$. By Remark 2.9, we have

$$
\xi \in F\left(P_{C}\left(I-\lambda_{n}(I-W)\right)\right)
$$

Observe that

$$
\begin{aligned}
\left\|W \phi_{n}-\xi\right\|^{2} & =\left\|\left(\phi_{n}-\xi\right)-(I-W) \phi_{n}\right\|^{2} \\
& =\left\|\phi_{n}-\xi\right\|^{2}-2\left\langle\phi_{n}-\xi,(I-W) \phi_{n}\right\rangle+\left\|(I-W) \phi_{n}\right\|^{2} \\
& \leq\left\|\phi_{n}-\xi\right\|^{2} .
\end{aligned}
$$

It implies that

$$
\left\|(I-W) \phi_{n}\right\|^{2} \leq 2\left\langle\phi_{n}-\xi,(I-W) \phi_{n}\right\rangle
$$

From (3.2) and (3.3), we have

$$
\begin{aligned}
\left\|P_{C}\left(I-\lambda_{n}(I-W)\right) \phi_{n}-\xi\right\|^{2}= & \left\|P_{C}\left(I-\lambda_{n}(I-W)\right) \phi_{n}-P_{C}\left(I-\lambda_{n}(I-W)\right) \xi\right\|^{2} \\
\leq & \left\|\left(\phi_{n}-\xi\right)-\lambda_{n}\left((I-W) \phi_{n}-(I-W) \xi\right)\right\|^{2} \\
= & \left\|\phi_{n}-\xi\right\|^{2}-2 \lambda_{n}\left(\phi_{n}-\xi,(I-W) \phi_{n}\right\rangle \\
& +\lambda_{n}^{2}\left\|(I-W) \phi_{n}\right\|^{2} \\
\leq & \left\|\phi_{n}-\xi\right\|^{2}+\lambda_{n}\left(\lambda_{n}-1\right)\left\|(I-W) \phi_{n}\right\|^{2} \\
\leq & \left\|\phi_{n}-\xi\right\|^{2} .
\end{aligned}
$$

From the definition of $p_{n}$ and (3.4), we have

$$
\begin{aligned}
\left\|p_{n+1}-\xi\right\|= & \| \alpha_{n}(u-\xi)+\beta_{n}\left(p_{n}-\xi\right)+\gamma_{n}\left(P_{C}\left(I-\lambda_{n}(I-W)\right) \phi_{n}-\xi\right) \\
& +\delta_{n}\left(G\left(\psi_{n}\right)-\xi\right) \| \\
\leq & \alpha_{n}\|u-\xi\|+\beta_{n}\left\|p_{n}-\xi\right\|+\gamma_{n}\left\|P_{C}\left(I-\lambda_{n}(I-W)\right) \phi_{n}-\xi\right\| \\
& +\delta_{n}\left\|G\left(\psi_{n}\right)-\xi\right\| \\
\leq & \alpha_{n}\|u-\xi\|+\beta_{n}\left\|p_{n}-\xi\right\|+\gamma_{n}\left\|\phi_{n}-\xi\right\|+\delta_{n}\left\|\psi_{n}-\xi\right\| \\
\leq & \alpha_{n}\|u-\xi\|+\beta_{n}\left\|p_{n}-\xi\right\|+\gamma_{n}\left\|p_{n}-\xi\right\|+\delta_{n}\left\|p_{n}-\xi\right\| \\
= & \alpha_{n}\|u-\xi\|+\left(1-\alpha_{n}\right)\left\|p_{n}-\xi\right\| .
\end{aligned}
$$

By induction, we can conclude that

$$
\left\|p_{n}-\xi\right\| \leq \max \left\{\|u-\xi\|,\left\|p_{1}-\xi\right\|\right\}
$$

for all $n \geq 1$. This implies that the sequence $\left\{p_{n}\right\}$ is bounded and so are $\left\{\phi_{n}\right\},\left\{\psi_{n}\right\},\{(I-$ $\left.W) \phi_{n}\right\}$, and $\left\{P_{C}\left(I-\lambda_{n}(I-W)\right) \phi_{n}\right\}$.

Then we show that $\lim _{n \rightarrow \infty}\left\|p_{n+1}-p_{n}\right\|=0$. 
From the definition of $p_{n}$ and nonexpansiveness of $G$, we have

$$
\begin{aligned}
\left\|p_{n+1}-p_{n}\right\|= & \|\left(\alpha_{n}-\alpha_{n-1}\right) u+\beta_{n}\left(p_{n}-p_{n-1}\right)+\left(\beta_{n}-\beta_{n-1}\right) p_{n-1} \\
& +\gamma_{n}\left(P_{C}\left(I-\lambda_{n}(I-W)\right) \phi_{n}-P_{C}\left(I-\lambda_{n-1}(I-W)\right) \phi_{n-1}\right) \\
& +\left(\gamma_{n}-\gamma_{n-1}\right) P_{C}\left(I-\lambda_{n-1}(I-W)\right) \phi_{n-1} \\
& +\delta_{n}\left(G\left(\psi_{n}\right)-G\left(\psi_{n-1}\right)\right)+\left(\delta_{n}-\delta_{n-1}\right) G\left(\psi_{n-1}\right) \| \\
\leq & \left|\alpha_{n}-\alpha_{n-1}\right|\|u\|+\beta_{n}\left\|p_{n}-p_{n-1}\right\|+\left|\beta_{n}-\beta_{n-1}\right|\left\|p_{n-1}\right\| \\
& +\gamma_{n}\left\|P_{C}\left(I-\lambda_{n}(I-W)\right) \phi_{n}-P_{C}\left(I-\lambda_{n-1}(I-W)\right) \phi_{n-1}\right\| \\
& +\left|\gamma_{n}-\gamma_{n-1}\right|\left\|P_{C}\left(I-\lambda_{n-1}(I-W)\right) \phi_{n-1}\right\| \\
& +\delta_{n}\left\|G\left(\psi_{n}\right)-G\left(\psi_{n-1}\right)\right\|+\left|\delta_{n}-\delta_{n-1}\right|\left\|G\left(\psi_{n-1}\right)\right\| \\
\leq & \left|\alpha_{n}-\alpha_{n-1}\right|\|u\|+\beta_{n}\left\|p_{n}-p_{n-1}\right\|+\left|\beta_{n}-\beta_{n-1}\right|\left\|p_{n-1}\right\| \\
& +\gamma_{n} \|\left(\phi_{n}-\phi_{n-1}\right)-\left(\lambda_{n}(I-W) \phi_{n}-\lambda_{n}(I-W) \phi_{n-1}\right) \\
& -\left(\lambda_{n}(I-W) \phi_{n-1}-\lambda_{n-1}(I-W) \phi_{n-1}\right) \| \\
& +\left|\gamma_{n}-\gamma_{n-1}\right|\left\|P_{C}\left(I-\lambda_{n-1}(I-W)\right) \phi_{n-1}\right\|+\delta_{n}\left\|\psi_{n}-\psi_{n-1}\right\| \\
& +\left|\delta_{n}-\delta_{n-1}\right|\left\|G\left(\psi_{n-1}\right)\right\| \\
\leq & \left|\alpha_{n}-\alpha_{n-1}\right|\|u\|+\beta_{n}\left\|p_{n}-p_{n-1}\right\|+\left|\beta_{n}-\beta_{n-1}\right|\left\|p_{n-1}\right\| \\
& +\gamma_{n}\left\|\phi_{n}-\phi_{n-1}\right\|+\lambda_{n}\left\|(I-W) \phi_{n}-(I-W) \phi_{n-1}\right\| \\
& +\left|\lambda_{n}-\lambda_{n-1}\right|\left\|(I-W) \phi_{n-1}\right\| \\
& +\left|\gamma_{n}-\gamma_{n-1}\right|\left\|P_{C}\left(I-\lambda_{n-1}(I-W)\right) \phi_{n-1}\right\|+\delta_{n}\left\|\psi_{n}-\psi_{n-1}\right\| \\
& +\left|\delta_{n}-\delta_{n-1}\right|\left\|G\left(\psi_{n-1}\right)\right\| . \\
&
\end{aligned}
$$

On the other hand, from $\phi_{n}=W_{g_{n}} p_{n}$ and $\phi_{n+1}=W_{g_{n+1}} p_{n+1}$, we have

$$
\Psi_{1}\left(\phi_{n}, \zeta\right)+\frac{1}{g_{n}}\left\langle\zeta-\phi_{n}, \phi_{n}-p_{n}\right\rangle \geq 0, \quad \forall \zeta \in C
$$

and

$$
\Psi_{1}\left(\phi_{n+1}, \zeta\right)+\frac{1}{g_{n+1}}\left\langle\zeta-\phi_{n+1}, \phi_{n+1}-p_{n+1}\right\rangle \geq 0, \quad \forall \zeta \in C
$$

Putting $\zeta=\phi_{n+1}$ in (3.6) and $\zeta=\phi_{n}$ in (3.7), we have

$$
\Psi_{1}\left(\phi_{n}, \phi_{n+1}\right)+\frac{1}{g_{n}}\left\langle\phi_{n+1}-\phi_{n}, \phi_{n}-p_{n}\right\rangle \geq 0
$$

and

$$
\Psi_{1}\left(\phi_{n+1}, \phi_{n}\right)+\frac{1}{g_{n+1}}\left\langle\phi_{n}-\phi_{n+1}, \phi_{n+1}-p_{n+1}\right\rangle \geq 0 .
$$


From (J2), we have

$$
\left\langle\phi_{n+1}-\phi_{n}, \frac{\phi_{n}-p_{n}}{g_{n}}-\frac{\phi_{n+1}-p_{n+1}}{g_{n+1}}\right\rangle \geq 0 .
$$

So

$$
\left\langle\phi_{n+1}-\phi_{n}, \phi_{n}-\phi_{n+1}+\phi_{n+1}-p_{n}-\frac{g_{n}}{g_{n+1}}\left(\phi_{n+1}-p_{n+1}\right)\right\rangle \geq 0
$$

Then

$$
\begin{aligned}
\left\|\phi_{n+1}-\phi_{n}\right\|^{2} & \leq\left\langle\phi_{n+1}-\phi_{n}, p_{n+1}-p_{n}+\phi_{n+1}-p_{n+1}-\frac{g_{n}}{g_{n+1}}\left(\phi_{n+1}-p_{n+1}\right)\right\rangle \\
& =\left\langle\phi_{n+1}-\phi_{n}, p_{n+1}-p_{n}+\left(1-\frac{g_{n}}{g_{n+1}}\right)\left(\phi_{n+1}-p_{n+1}\right)\right\rangle \\
& \leq\left\|\phi_{n+1}-\phi_{n}\right\|\left(\left\|p_{n+1}-p_{n}\right\|+\left|1-\frac{g_{n}}{g_{n+1}}\right|\left\|\phi_{n+1}-p_{n+1}\right\|\right),
\end{aligned}
$$

and hence

$$
\begin{aligned}
\left\|\phi_{n+1}-\phi_{n}\right\| & \leq\left\|p_{n+1}-p_{n}\right\|+\frac{1}{g_{n+1}}\left|g_{n+1}-g_{n}\right|\left\|\phi_{n+1}-p_{n+1}\right\| \\
& \leq\left\|p_{n+1}-p_{n}\right\|+\frac{1}{e}\left|g_{n+1}-g_{n}\right|\left\|\phi_{n+1}-p_{n+1}\right\| .
\end{aligned}
$$

We use $\psi_{n}=W_{h_{n}} p_{n}$ and $\psi_{n+1}=W_{h_{n+1}} p_{n+1}$. By using the same method as (3.8), we have

$$
\left\|\psi_{n+1}-\psi_{n}\right\| \leq\left\|p_{n+1}-p_{n}\right\|+\frac{1}{e}\left|h_{n+1}-h_{n}\right|\left\|\psi_{n+1}-p_{n+1}\right\| .
$$

From (3.5), (3.8), and (3.9), we have

$$
\begin{aligned}
\left\|p_{n+1}-p_{n}\right\| \leq & \left|\alpha_{n}-\alpha_{n-1}\right|\|u\|+\beta_{n}\left\|p_{n}-p_{n-1}\right\|+\left|\beta_{n}-\beta_{n-1}\right|\left\|p_{n-1}\right\| \\
& +\gamma_{n}\left(\left\|p_{n+1}-p_{n}\right\|+\frac{1}{e}\left|g_{n+1}-g_{n}\right|\left\|\phi_{n+1}-p_{n+1}\right\|\right) \\
& +\lambda_{n}\left\|(I-W) \phi_{n}-(I-W) \phi_{n-1}\right\|+\left|\lambda_{n}-\lambda_{n-1}\right|\left\|(I-W) \phi_{n-1}\right\| \\
& +\left|\gamma_{n}-\gamma_{n-1}\right|\left\|P_{C}\left(I-\lambda_{n-1}(I-W)\right) \phi_{n-1}\right\| \\
& +\delta_{n}\left(\left\|p_{n+1}-p_{n}\right\|+\frac{1}{e}\left|h_{n+1}-h_{n}\right|\left\|\psi_{n+1}-p_{n+1}\right\|\right) \\
& +\left|\delta_{n}-\delta_{n-1}\right|\left\|G\left(\psi_{n-1}\right)\right\| \\
\leq & \left|\alpha_{n}-\alpha_{n-1}\right|\|u\|+\beta_{n}\left\|p_{n}-p_{n-1}\right\|+\left|\beta_{n}-\beta_{n-1}\right|\left\|p_{n-1}\right\| \\
& +\gamma_{n}\left\|p_{n+1}-p_{n}\right\|+\frac{1}{e}\left|g_{n+1}-g_{n}\right|\left\|\phi_{n+1}-p_{n+1}\right\| \\
& +\lambda_{n}\left\|(I-W) \phi_{n}-(I-W) \phi_{n-1}\right\|+\left|\lambda_{n}-\lambda_{n-1}\right|\left\|(I-W) \phi_{n-1}\right\| \\
& +\left|\gamma_{n}-\gamma_{n-1}\right|\left\|P_{C}\left(I-\lambda_{n-1}(I-W)\right) \phi_{n-1}\right\|+\delta_{n}\left\|p_{n+1}-p_{n}\right\| \\
& +\frac{1}{e}\left|h_{n+1}-h_{n}\right|\left\|\psi_{n+1}-p_{n+1}\right\|+\left|\delta_{n}-\delta_{n-1}\right|\left\|G\left(\psi_{n-1}\right)\right\|
\end{aligned}
$$




$$
\begin{aligned}
\leq & \left(1-\alpha_{n}\right)|| p_{n}-p_{n-1}||+\left|\alpha_{n}-\alpha_{n-1}\right| M+\left|\beta_{n}-\beta_{n-1}\right| M \\
& +\left|\gamma_{n}-\gamma_{n-1}\right| M+\left|\delta_{n}-\delta_{n-1}\right| M+\left|\lambda_{n}-\lambda_{n-1}\right| M+\lambda_{n} M \\
& +\frac{1}{e}\left|g_{n+1}-g_{n}\right| M+\frac{1}{e}\left|h_{n+1}-h_{n}\right| M,
\end{aligned}
$$

where

$$
\begin{aligned}
M:= & \max _{n \in \mathbb{N}}\left\{\|u\|,\left\|p_{n}\right\|,\left\|P_{C}\left(I-\lambda_{n}(I-W)\right) \phi_{n}\right\|,\left\|G\left(\psi_{n}\right)\right\|,\left\|(I-W) \phi_{n}\right\|,\right. \\
& \left.\left\|(I-W) \phi_{n+1}-(I-W) \phi_{n}\right\|,\left\|\phi_{n}-p_{n}\right\|,\left\|\psi_{n}-p_{n}\right\|\right\} .
\end{aligned}
$$

From the conditions (i), (iv), (v), and Lemma 2.6, we have

$$
\lim _{n \rightarrow \infty}\left\|p_{n+1}-p_{n}\right\|=0
$$

Since $W_{g_{n}}$ is a firmly nonexpansive mapping, we obtain

$$
\begin{aligned}
\left\|\phi_{n}-\xi\right\|^{2} & =\left\|W_{g_{n}} p_{n}-W_{g_{n}} \xi\right\|^{2} \\
& \leq\left\langle W_{g_{n}} p_{n}-W_{g_{n}} \xi, p_{n}-\xi\right\rangle \\
& \leq\left\langle\phi_{n}-\xi, p_{n}-\xi\right\rangle \\
& =\frac{1}{2}\left(\left\|\phi_{n}-\xi\right\|^{2}+\left\|p_{n}-\xi\right\|^{2}-\left\|\phi_{n}-p_{n}\right\|^{2}\right) .
\end{aligned}
$$

It implies that

$$
\left\|\phi_{n}-\xi\right\|^{2} \leq\left\|p_{n}-\xi\right\|^{2}-\left\|\phi_{n}-p_{n}\right\|^{2}
$$

By using the same method as (3.11), we have

$$
\left\|\psi_{n}-\xi\right\|^{2} \leq\left\|p_{n}-\xi\right\|^{2}-\left\|\psi_{n}-p_{n}\right\|^{2}
$$

From the definition of $p_{n},(3.4),(3.11)$, and (3.12), we have

$$
\begin{aligned}
\left\|p_{n+1}-\xi\right\|^{2}= & \| \alpha_{n}(u-\xi)+\beta_{n}\left(p_{n}-\xi\right)+\gamma_{n}\left(P_{C}\left(I-\lambda_{n}(I-W)\right) \phi_{n}-\xi\right) \\
& +\delta_{n}\left(G\left(\psi_{n}\right)-\xi\right) \|^{2} \\
\leq & \alpha_{n}\|u-\xi\|^{2}+\beta_{n}\left\|p_{n}-\xi\right\|^{2}+\gamma_{n}\left\|P_{C}\left(I-\lambda_{n}(I-W)\right) \phi_{n}-\xi\right\|^{2} \\
& +\delta_{n}\left\|G\left(\psi_{n}\right)-\xi\right\|^{2}-\beta_{n} \gamma_{n}\left\|P_{C}\left(I-\lambda_{n}(I-W)\right) \phi_{n}-p_{n}\right\|^{2} \\
& -\beta_{n} \delta_{n}\left\|G\left(\psi_{n}\right)-p_{n}\right\|^{2} \\
\leq & \alpha_{n}\|u-\xi\|^{2}+\beta_{n}\left\|p_{n}-\xi\right\|^{2}+\gamma_{n}\left\|\phi_{n}-\xi\right\|^{2}+\delta_{n}\left\|\psi_{n}-\xi\right\|^{2} \\
& \quad-\beta_{n} \gamma_{n}\left\|P_{C}\left(I-\lambda_{n}(I-W)\right) \phi_{n}-p_{n}\right\|^{2}-\beta_{n} \delta_{n}\left\|G\left(\psi_{n}\right)-p_{n}\right\|^{2} \\
\leq & \alpha_{n}\|u-\xi\|^{2}+\beta_{n}\left\|p_{n}-\xi\right\|^{2}+\gamma_{n}\left(\left\|p_{n}-\xi\right\|^{2}-\left\|\phi_{n}-p_{n}\right\|^{2}\right) \\
& +\delta_{n}\left(\left\|p_{n}-\xi\right\|^{2}-\left\|\psi_{n}-p_{n}\right\|^{2}\right)-\beta_{n} \delta_{n}\left\|G\left(\psi_{n}\right)-p_{n}\right\|^{2}
\end{aligned}
$$




$$
\begin{aligned}
& -\beta_{n} \gamma_{n}\left\|P_{C}\left(I-\lambda_{n}(I-W)\right) \phi_{n}-p_{n}\right\|^{2} \\
= & \alpha_{n}\|u-\xi\|^{2}+\left(1-\alpha_{n}\right)\left\|p_{n}-\xi\right\|^{2}-\gamma_{n}\left\|\phi_{n}-p_{n}\right\|^{2} \\
& -\delta_{n}\left\|\psi_{n}-p_{n}\right\|^{2}-\beta_{n} \gamma_{n}\left\|P_{C}\left(I-\lambda_{n}(I-W)\right) \phi_{n}-p_{n}\right\|^{2} \\
& -\beta_{n} \delta_{n}\left\|G\left(\psi_{n}\right)-p_{n}\right\|^{2} \\
\leq & \alpha_{n}\|u-\xi\|^{2}+\left\|p_{n}-\xi\right\|^{2}-\gamma_{n}\left\|\phi_{n}-p_{n}\right\|^{2}-\delta_{n}\left\|\psi_{n}-p_{n}\right\|^{2} \\
& -\beta_{n} \gamma_{n}\left\|P_{C}\left(I-\lambda_{n}(I-W)\right) \phi_{n}-p_{n}\right\|^{2}-\beta_{n} \delta_{n}\left\|G\left(\psi_{n}\right)-p_{n}\right\|^{2},
\end{aligned}
$$

which implies that

$$
\begin{aligned}
\gamma_{n}\left\|\phi_{n}-p_{n}\right\|^{2} & \leq \alpha_{n}\|u-\xi\|^{2}+\left\|p_{n}-\xi\right\|^{2}-\left\|p_{n+1}-\xi\right\|^{2} \\
& \leq \alpha_{n}\|u-\xi\|^{2}+\left\|p_{n}-p_{n+1}\right\|\left(\left\|p_{n}-\xi\right\|+\left\|p_{n+1}-\xi\right\|\right) .
\end{aligned}
$$

From the conditions (i), (ii), and (3.10), we have

$$
\lim _{n \rightarrow \infty}\left\|\phi_{n}-p_{n}\right\|=0
$$

By using the same method as (3.13), we can imply that

$$
\lim _{n \rightarrow \infty}\left\|\psi_{n}-p_{n}\right\|=\lim _{n \rightarrow \infty}\left\|P_{C}\left(I-\lambda_{n}(I-W)\right) \phi_{n}-p_{n}\right\|=\lim _{n \rightarrow \infty}\left\|G\left(\psi_{n}\right)-p_{n}\right\|=0 .
$$

From (3.13) and (3.14), we have

$$
\lim _{n \rightarrow \infty}\left\|\phi_{n}-\psi_{n}\right\|=0
$$

Afterwards, we show that $\lim \sup _{n \rightarrow \infty}\left\langle u-p_{0}, p_{n}-p_{0}\right\rangle \leq 0$, where $p_{0}=P_{\mathcal{F}} u$. To show this inequality, take a subsequence $\left\{p_{n_{j}}\right\}$ of $\left\{p_{n}\right\}$ such that

$$
\limsup _{n \rightarrow \infty}\left\langle u-p_{0}, p_{n}-p_{0}\right\rangle=\lim _{j \rightarrow \infty}\left\langle u-p_{0}, p_{n_{j}}-p_{0}\right\rangle .
$$

Without loss of generality, we may assume that $u_{n_{j}} \rightarrow \omega$ as $j \rightarrow \infty$. From (3.15), we have $v_{n_{j}} \rightarrow \omega$ as $j \rightarrow \infty$. By using the same method as [15] in Theorem 3.2, we have

$$
\omega \in \operatorname{EP}\left(\Psi_{1}\right)
$$

and

$$
\omega \in \operatorname{EP}\left(\Psi_{2}\right)
$$

Furthermore, we show that $\omega \in F(W)$. From Remark 2.9, we have $F(W)=F\left(P_{C}\left(I-\lambda_{n_{j}}(I-\right.\right.$ $W))$ ). Assume that $\omega \notin F(W)$, we have $\omega \neq P_{C}\left(I-\lambda_{n_{j}}(I-W)\right) \omega$. From (3.13), we have $p_{n_{j}} \rightarrow$ $\omega$ as $j \rightarrow \infty$. By (3.13), (3.14), the condition (iv), and Opial's property, we have

$$
\begin{aligned}
\liminf _{j \rightarrow \infty}\left\|p_{n_{j}}-\omega\right\| & <\liminf _{j \rightarrow \infty}\left\|p_{n_{j}}-P_{C}\left(I-\lambda_{n_{j}}(I-W)\right) \omega\right\| \\
& \leq \liminf _{j \rightarrow \infty}\left(\left\|p_{n_{j}}-P_{C}\left(I-\lambda_{n_{j}}(I-W)\right) u_{n_{j}}\right\|\right.
\end{aligned}
$$




$$
\begin{aligned}
& +\left\|P_{C}\left(I-\lambda_{n_{j}}(I-W)\right) u_{n_{j}}-P_{C}\left(I-\lambda_{n_{j}}(I-W)\right) p_{n_{j}}\right\| \\
& \left.+\left\|P_{C}\left(I-\lambda_{n_{j}}(I-W)\right) p_{n_{j}}-P_{C}\left(I-\lambda_{n_{j}}(I-W)\right) \omega\right\|\right) \\
\leq & \liminf _{j \rightarrow \infty}\left(\left\|u_{n_{j}}-p_{n_{j}}\right\|+\lambda_{n_{j}}\left\|(I-W) u_{n_{j}}-(I-W) p_{n_{j}}\right\|\right. \\
& \left.+\left\|p_{n_{j}}-\omega\right\|+\lambda_{n_{j}}\left\|(I-W) p_{n_{j}}-(I-W) \omega\right\|\right) \\
= & \liminf _{j \rightarrow \infty}\left\|p_{n_{j}}-\omega\right\| .
\end{aligned}
$$

It is a contradiction. So we have

$$
\omega \in F(W) .
$$

After that, we show that $\omega \in F(G)$. Assume that $\omega \notin F(G)$, that is, $\omega \neq G(\omega)$. Since $p_{n_{j}} \rightarrow$ $\omega$ as $j \rightarrow \infty,(3.14)$, the condition (iv), and Opial's property, we have

$$
\begin{aligned}
\liminf _{j \rightarrow \infty}\left\|p_{n_{j}}-\omega\right\|< & \liminf _{j \rightarrow \infty}\left\|p_{n_{j}}-G(\omega)\right\| \\
\leq & \liminf _{j \rightarrow \infty}\left(\left\|p_{n_{j}}-G\left(\psi_{n_{j}}\right)\right\|+\left\|G\left(\psi_{n_{j}}\right)-G\left(p_{n_{j}}\right)\right\|\right. \\
& \left.+\left\|G\left(p_{n_{j}}\right)-G(\omega)\right\|\right) \\
\leq & \liminf _{j \rightarrow \infty}\left(\left\|\psi_{n_{j}}-p_{n_{j}}\right\|+\left\|p_{n_{j}}-\omega\right\|\right) \\
= & \liminf _{j \rightarrow \infty}\left\|p_{n_{j}}-\omega\right\| .
\end{aligned}
$$

It is a contradiction. So we have

$$
\omega \in F(G)
$$

Therefore $\omega \in \mathcal{F}$. Since $p_{n_{j}} \rightarrow \omega$ as $j \rightarrow \infty$, we have

$$
\begin{aligned}
\limsup _{n \rightarrow \infty}\left\langle u-p_{0}, p_{n}-p_{0}\right\rangle & =\lim _{j \rightarrow \infty}\left\langle u-p_{0}, p_{n_{j}}-p_{0}\right\rangle \\
& =\left\langle u-p_{0}, \omega-p_{0}\right\rangle \leq 0 .
\end{aligned}
$$

Finally, we show that the sequences $\left\{p_{n}\right\},\left\{\phi_{n}\right\}$, and $\left\{\psi_{n}\right\}$ converge strongly to $p_{0}=P_{\mathcal{F}} u$. From the definition of $p_{n},(3.4)$, and $p_{0}=P_{\mathcal{F}} u$, we have

$$
\begin{aligned}
\left\|p_{n+1}-p_{0}\right\|^{2}= & \| \alpha_{n}\left(u-p_{0}\right)+\beta_{n}\left(p_{n}-p_{0}\right)+\gamma_{n}\left(P_{C}\left(I-\lambda_{n}(I-W)\right) \phi_{n}-p_{0}\right) \\
& +\delta_{n}\left(G\left(\psi_{n}\right)-p_{0}\right) \|^{2} \\
\leq & \| \beta_{n}\left(p_{n}-p_{0}\right)+\gamma_{n}\left(P_{C}\left(I-\lambda_{n}(I-W)\right) \phi_{n}-p_{0}\right) \\
& +\delta_{n}\left(G\left(\psi_{n}\right)-p_{0}\right) \|^{2}+2 \alpha_{n}\left\langle u-p_{0}, p_{n+1}-p_{0}\right\rangle \\
\leq & \left(1-\alpha_{n}\right)\left\|p_{n}-p_{0}\right\|^{2}+2 \alpha_{n}\left\langle u-p_{0}, p_{n+1}-p_{0}\right\rangle .
\end{aligned}
$$

From the condition (i), (3.20), and Lemma 2.6, we can conclude that the sequence $\left\{p_{n}\right\}$ converges strongly to $p_{0}=P_{\mathcal{F}} u$. Consequently, we see that $\left\{\phi_{n}\right\}$ and $\left\{\psi_{n}\right\}$ also converge strongly to $p_{0}=P_{\mathcal{F}} u$. This completes the proof. 
From our main result, if we take $a=0$, we have the following corollary.

Corollary 3.2 Let $C$ be a nonempty closed convex subset of a real Hilbert space $H$, let $\Psi_{1}, \Psi_{2}: C \times C \rightarrow \mathbb{R}$ be bifunctions satisfying (J1)-(J4) and let $W: C \rightarrow C$ be a quasinonexpansive mapping. Let $D_{1}, D_{2}: C \rightarrow H$ be $d_{1}, d_{2}$-inverse strongly monotone mappings, respectively. Define the mapping $G: C \rightarrow C$ by $G(p)=P_{C}\left(I-\lambda_{A} D_{1}\right)\left(P_{C}\left(I-\lambda_{B} D_{2}\right) p\right)$ for all $p \in C$. Assume $\mathcal{F}=\operatorname{EP}\left(\Psi_{1}\right) \cap \operatorname{EP}\left(\Psi_{2}\right) \cap F(G) \cap F(W) \neq \emptyset$. Suppose that $p_{1}, u \in C$ and let $\left\{p_{n}\right\},\left\{\phi_{n}\right\}$, and $\left\{\psi_{n}\right\}$ be sequences generated by

$$
\left\{\begin{array}{l}
\Psi_{1}\left(\phi_{n}, \zeta\right)+\frac{1}{g_{n}}\left\langle\zeta-\phi_{n}, \phi_{n}-p_{n}\right\rangle \geq 0, \quad \forall \zeta \in C, \\
\Psi_{2}\left(\psi_{n}, \zeta\right)+\frac{1}{h_{n}}\left\langle\zeta-\psi_{n}, \psi_{n}-p_{n}\right\rangle \geq 0, \quad \forall \zeta \in C, \\
p_{n+1}=\alpha_{n} u+\beta_{n} p_{n}+\gamma_{n} P_{C}\left(I-\lambda_{n}(I-W)\right) \phi_{n}+\delta_{n} G\left(\psi_{n}\right), \quad \forall n \in \mathbb{N},
\end{array}\right.
$$

where the sequences $\lambda_{A} \in\left(0,2 d_{1}\right), \lambda_{B} \in\left(0,2 d_{2}\right)$ and $\left\{\alpha_{n}\right\},\left\{\beta_{n}\right\},\left\{\gamma_{n}\right\},\left\{\delta_{n}\right\} \subseteq[0,1]$ with $\alpha_{n}+$ $\beta_{n}+\gamma_{n}+\delta_{n}=1$ for all $n \in \mathbb{N}$. Suppose the following conditions hold:

(i) $\lim _{n \rightarrow \infty} \alpha_{n}=0$ and $\sum_{n=1}^{\infty} \alpha_{n}=\infty$,

(ii) $0<c \leq \beta_{n}, \gamma_{n}, \delta_{n} \leq d<1$ for some $c, d>0$ and for all $n \geq 1$,

(iii) $0<e \leq g_{n}, h_{n} \leq$ for some $e, f>0$ and for all $n \geq 1$,

(iv) $\sum_{n=1}^{\infty} \lambda_{n}<\infty$ and $0<\lambda_{n}<1$,

(v) $\sum_{n=1}^{\infty}\left|\alpha_{n+1}-\alpha_{n}\right|<\infty, \sum_{n=1}^{\infty}\left|\beta_{n+1}-\beta_{n}\right|<\infty, \sum_{n=1}^{\infty}\left|\gamma_{n+1}-\gamma_{n}\right|<\infty$, $\sum_{n=1}^{\infty}\left|\lambda_{n+1}-\lambda_{n}\right|<\infty, \sum_{n=1}^{\infty}\left|g_{n+1}-g_{n}\right|<\infty, \sum_{n=1}^{\infty}\left|h_{n+1}-h_{n}\right|<\infty$.

Then $\left\{p_{n}\right\},\left\{\phi_{n}\right\}$, and $\left\{\psi_{n}\right\}$ converge strongly to $p_{0}=P_{\mathcal{F}} u$ and $\left(p_{0}, \xi_{0}\right)$ is a solution of (1.5) where $\xi_{0}=P_{C}\left(I-\lambda_{B} D_{2}\right) p_{0}$.

\section{Application}

In this section, we use our main result to obtain Theorem 4.7 and Theorem 4.8. Before we prove these theorems, we need the following definition and lemma. A mapping $W: C \rightarrow C$ is said to be nonspreading if

$$
2\|W p-W \zeta\|^{2} \leq\|W p-\zeta\|^{2}+\|W \zeta-p\|^{2}, \quad \forall p, \zeta \in C
$$

Such a mapping is defined by Kohsaka and Takahashi [16].

In 2009, Iemoto and Takahashi [17] proved that (4.1) is equivalent to

$$
\|W p-W \zeta\|^{2} \leq\|p-\zeta\|^{2}+2\langle p-W p, \zeta-W \zeta\rangle, \quad \forall p, \zeta \in C .
$$

Remark 4.1 A nonspreading mapping $W$ with $F(W) \neq \emptyset$ is quasi-nonexpansive mapping.

Example 4.2 Let $W:[-5, \infty) \rightarrow[-5, \infty)$ be defined by

$$
W p=\frac{p-5}{2}, \quad \forall p \in[-5, \infty)
$$

Since $W$ is a nonspreading mapping and $F(W)=\{-5\}$, we have $W$ is a quasi-nonexpansive mapping.

The following lemmas and definition are used to prove the results in this section. 
Lemma 4.3 ([8]) Let $C$ be a nonempty closed convex subset of a real Hilbert space $H$ and let $D_{1}, D_{2}: C \rightarrow H$ be $d_{1}, d_{2}$-inverse strongly monotone mappings, respectively, with $\operatorname{VIP}\left(C, D_{1}\right) \cap \operatorname{VIP}\left(C, D_{2}\right) \neq \emptyset$. Define a mapping $G: C \rightarrow C$ by

$$
G(p)=P_{C}\left(I-\lambda_{A} D_{1}\right)\left(a p+(1-a) P_{C}\left(I-\lambda_{B} D_{2}\right) p\right),
$$

for every $\lambda_{A} \in\left(0,2 d_{1}\right), \lambda_{B} \in\left(0,2 d_{2}\right)$ and $a \in(0,1)$. Then $F(G)=\operatorname{VIP}\left(C, D_{1}\right) \cap \operatorname{VIP}\left(C, D_{2}\right)$.

Lemma 4.4 ([16]) Let $H$ be a Hilbert space, let $C$ be a nonempty closed convex subset of $H$, and let $W$ be a nonspreading mapping of $C$ into itself. Then $F(W)$ is closed and convex.

In 2009, Kangtunyakarn and Suantai [18] introduced the $S$-mapping generated by $W_{1}, W_{2}, W_{3}, \ldots, W_{N}$ and $\lambda_{1}, \lambda_{2}, \ldots, \lambda_{N}$ as follows.

Definition 4.5 Let $C$ be a nonempty convex subset of a real Banach space. Let $\left\{W_{i}\right\}_{i=1}^{N}$ be a finite family of (nonexpansive) mappings of $C$ into itself. For each $j=1,2, \ldots, N$, let $\alpha_{j}=$ $\left(\alpha_{1}^{j}, \alpha_{2}^{j}, \alpha_{3}^{j}\right) \in I \times I \times I$, where $I \in[0,1]$ and $\alpha_{1}^{j}+\alpha_{2}^{j}+\alpha_{3}^{j}=1$. Define the mapping $S: C \rightarrow C$ as follows:

$$
\begin{aligned}
& U_{0}=I, \\
& U_{1}=\alpha_{1}^{1} W_{1} U_{0}+\alpha_{2}^{1} U_{0}+\alpha_{3}^{1} I, \\
& U_{2}=\alpha_{1}^{2} W_{2} U_{1}+\alpha_{2}^{2} U_{1}+\alpha_{3}^{2} I, \\
& U_{3}=\alpha_{1}^{3} W_{3} U_{2}+\alpha_{2}^{3} U_{2}+\alpha_{3}^{3} I, \\
& \ldots, \\
& U_{N-1}=\alpha_{1}^{N-1} W_{N-1} U_{N-2}+\alpha_{2}^{N-1} U_{N-2}+\alpha_{3}^{N-1} I, \\
& S=U_{N}=\alpha_{1}^{N} W_{N} U_{N-1}+\alpha_{2}^{N} U_{N-1}+\alpha_{3}^{N} I .
\end{aligned}
$$

This mapping is called an $S$-mapping generated by $W_{1}, W_{2}, \ldots, W_{N}$ and $\alpha_{1}, \alpha_{2}, \ldots, \alpha_{N}$.

For every $i=1,2, \ldots N$, put $\alpha_{3}^{i}=0$ in Definition 4.5, then the $S$-mapping is reduced to the $K$-mapping generated by $\alpha_{1}^{1}, \alpha_{1}^{2}, \ldots, \alpha_{1}^{N}$ where the $K$-mapping is defined by Kangtunyakarn and Suantai [19] as follows.

Lemma 4.6 ([20]) Let $C$ be a nonempty closed convex subset of a real Hilbert space. Let $\left\{W_{i}\right\}_{i=1}^{N}$ be a finite family of nonspreading mappings of $C$ into $C$ with $\bigcap_{i=1}^{N} F\left(W_{i}\right) \neq \emptyset$, and let $\alpha_{j}=\left(\alpha_{1}^{j}, \alpha_{2}^{j}, \alpha_{3}^{j}\right) \in I \times I \times I, j=1,2, \ldots, N$, where $I=[0,1], \alpha_{1}^{j}+\alpha_{2}^{j}+\alpha_{3}^{j}=1, \alpha_{1}^{j}, \alpha_{3}^{j} \in(0,1)$ for all $j=1,2, \ldots, N-1$ and $\alpha_{1}^{N} \in(0,1], \alpha_{3}^{N} \in[0,1), \alpha_{2}^{j} \in[0,1)$ for all $j=1,2, \ldots, N$. Let $S$ be the mapping generated by $W_{1}, W_{2}, \ldots, W_{N}$ and $\alpha_{1}, \alpha_{2}, \ldots, \alpha_{N}$. Then $F(S)=\bigcap_{i=1}^{N} F\left(W_{i}\right)$ and $S$ is a quasi-nonexpansive mapping.

By using these results, we obtain the following theorems.

Theorem 4.7 Let $C$ be a nonempty closed convex subset of a real Hilbert space $H$, let $\Psi_{1}, \Psi_{2}: C \times C \rightarrow \mathbb{R}$ be bifunctions satisfying (J1)-(J4) and let $W: C \rightarrow C$ be a quasinonexpansive mapping. Let $D_{1}, D_{2}: C \rightarrow H$ be $d_{1}, d_{2}$-inverse strongly monotone mappings, 
respectively. Assume $\mathcal{F}=\mathrm{EP}\left(\Psi_{1}\right) \cap \mathrm{EP}\left(\Psi_{2}\right) \cap F(W) \cap \operatorname{VIP}\left(C, D_{1}\right) \cap \operatorname{VIP}\left(C, D_{2}\right) \neq \emptyset$. Suppose that $p_{1}, u \in C$ and let $\left\{p_{n}\right\},\left\{\phi_{n}\right\}$, and $\left\{\psi_{n}\right\}$ be sequences generated by

$$
\left\{\begin{array}{l}
\Psi_{1}\left(\phi_{n}, \zeta\right)+\frac{1}{g_{n}}\left\langle\zeta-\phi_{n}, \phi_{n}-p_{n}\right\rangle \geq 0, \quad \forall \zeta \in C, \\
\Psi_{2}\left(\psi_{n}, \zeta\right)+\frac{1}{h_{n}}\left\langle\zeta-\psi_{n}, \psi_{n}-p_{n}\right\rangle \geq 0, \quad \forall \zeta \in C, \\
p_{n+1}=\alpha_{n} u+\beta_{n} p_{n}+\gamma_{n} P_{C}\left(I-\lambda_{n}(I-W)\right) \phi_{n} \\
\quad+\delta_{n} P_{C}\left(I-\lambda_{A} D_{1}\right)\left(a p_{n}+(1-a) P_{C}\left(I-\lambda_{B} D_{2}\right) p_{n}\right), \quad \forall n \in \mathbb{N},
\end{array}\right.
$$

where the sequences $\lambda_{A} \in\left(0,2 d_{1}\right), \lambda_{B} \in\left(0,2 d_{2}\right)$, and $\left\{\alpha_{n}\right\},\left\{\beta_{n}\right\},\left\{\gamma_{n}\right\},\left\{\delta_{n}\right\} \subseteq[0,1]$ with $\alpha_{n}+$ $\beta_{n}+\gamma_{n}+\delta_{n}=1$, for all $n \in \mathbb{N}$, and $a \in(0,1)$. Suppose the following conditions hold:

(i) $\lim _{n \rightarrow \infty} \alpha_{n}=0$ and $\sum_{n=1}^{\infty} \alpha_{n}=\infty$,

(ii) $0<c \leq \beta_{n}, \gamma_{n}, \delta_{n} \leq d<1$ for some $c, d>0$ and for all $n \geq 1$,

(iii) $0<e \leq g_{n}, h_{n} \leq f$ for some $e, f>0$ and for all $n \geq 1$,

(iv) $\sum_{n=1}^{\infty} \lambda_{n}<\infty$ and $0<\lambda_{n}<1$,

(v) $\sum_{n=1}^{\infty}\left|\alpha_{n+1}-\alpha_{n}\right|<\infty, \sum_{n=1}^{\infty}\left|\beta_{n+1}-\beta_{n}\right|<\infty, \sum_{n=1}^{\infty}\left|\gamma_{n+1}-\gamma_{n}\right|<\infty$, $\sum_{n=1}^{\infty}\left|\lambda_{n+1}-\lambda_{n}\right|<\infty, \sum_{n=1}^{\infty}\left|g_{n+1}-g_{n}\right|<\infty, \sum_{n=1}^{\infty}\left|h_{n+1}-h_{n}\right|<\infty$.

Then $\left\{p_{n}\right\}$, $\left\{\phi_{n}\right\}$, and $\left\{\psi_{n}\right\}$ converge strongly to $p_{0}=P_{\mathcal{F}} u$ and $\left(p_{0}, \xi_{0}\right)$ be a solution of (1.6) where $\xi_{0}=P_{C}\left(I-\lambda_{B} D_{2}\right) p_{0}$.

Proof By using Theorem 3.1 and Lemma 4.3, we obtain the conclusion.

Theorem 4.8 Let $C$ be a nonempty closed convex subset of a real Hilbert space $H$, let $\Psi_{1}, \Psi_{2}: C \times C \rightarrow \mathbb{R}$ be bifunctions satisfying (J1)-(J4). Let $\left\{W_{i}\right\}_{i=1}^{N}$ be a finite family of nonspreading mappings of $C$ into $C$ and let $\alpha_{j}=\left(\alpha_{1}^{j}, \alpha_{2}^{j}, \alpha_{3}^{j}\right) \in I \times I \times I, j=1,2, \ldots, N$, where $I=[0,1], \alpha_{1}^{j}+\alpha_{2}^{j}+\alpha_{3}^{j}=1, \alpha_{1}^{j}, \alpha_{3}^{j} \in(0,1)$ for all $j=1,2, \ldots, N-1$ and $\alpha_{1}^{N} \in(0,1], \alpha_{3}^{N} \in[0,1)$, $\alpha_{2}^{j} \in[0,1)$ for all $j=1,2, \ldots, N$. Let $S$ be the mapping generated by $W_{1}, W_{2}, \ldots, W_{N}$, and $\alpha_{1}, \alpha_{2}, \ldots, \alpha_{N}$. Let $D_{1}, D_{2}: C \rightarrow H$ be $d_{1}, d_{2}$-inverse strongly monotone mappings, respectively. Define the mapping $G: C \rightarrow C$ by $G(p)=P_{C}\left(I-\lambda_{A} D_{1}\right)\left(a p+(1-a) P_{C}\left(I-\lambda_{B} D_{2}\right) p\right)$ for all $p \in C$ and $a \in[0,1]$. Assume $\mathcal{F}=\mathrm{EP}\left(\Psi_{1}\right) \cap \operatorname{EP}\left(\Psi_{2}\right) \cap F(G) \cap \bigcap_{i=1}^{N} F\left(W_{i}\right) \neq \emptyset$. Suppose that $p_{1}, u \in C$ and let $\left\{p_{n}\right\},\left\{\phi_{n}\right\}$, and $\left\{\psi_{n}\right\}$ are sequences generated by

$$
\left\{\begin{array}{l}
\Psi_{1}\left(\phi_{n}, \zeta\right)+\frac{1}{g_{n}}\left\langle\zeta-\phi_{n}, \phi_{n}-p_{n}\right\rangle \geq 0, \quad \forall \zeta \in C, \\
\Psi_{2}\left(\psi_{n}, \zeta\right)+\frac{1}{h_{n}}\left\langle\zeta-\psi_{n}, \psi_{n}-p_{n}\right\rangle \geq 0, \quad \forall \zeta \in C, \\
p_{n+1}=\alpha_{n} u+\beta_{n} p_{n}+\gamma_{n} P_{C}\left(I-\lambda_{n}(I-S)\right) \phi_{n}+\delta_{n} G\left(\psi_{n}\right), \quad \forall n \in \mathbb{N},
\end{array}\right.
$$

where the sequences $\lambda_{A} \in\left(0,2 d_{1}\right), \lambda_{B} \in\left(0,2 d_{2}\right)$, and $\left\{\alpha_{n}\right\},\left\{\beta_{n}\right\},\left\{\gamma_{n}\right\},\left\{\delta_{n}\right\} \subseteq[0,1]$ with $\alpha_{n}+$ $\beta_{n}+\gamma_{n}+\delta_{n}=1$ for all $n \in \mathbb{N}$. Suppose the following conditions hold:

(i) $\lim _{n \rightarrow \infty} \alpha_{n}=0$ and $\sum_{n=1}^{\infty} \alpha_{n}=\infty$,

(ii) $0<c \leq \beta_{n}, \gamma_{n}, \delta_{n} \leq d<1$ for some $c, d>0$ and for all $n \geq 1$,

(iii) $0<e \leq g_{n}, h_{n} \leq f$ for some $e, f>0$ and for all $n \geq 1$,

(iv) $\sum_{n=1}^{\infty} \lambda_{n}<\infty$ and $0<\lambda_{n}<1$,

(v) $\sum_{n=1}^{\infty}\left|\alpha_{n+1}-\alpha_{n}\right|<\infty, \sum_{n=1}^{\infty}\left|\beta_{n+1}-\beta_{n}\right|<\infty, \sum_{n=1}^{\infty}\left|\gamma_{n+1}-\gamma_{n}\right|<\infty$, $\sum_{n=1}^{\infty}\left|\lambda_{n+1}-\lambda_{n}\right|<\infty, \sum_{n=1}^{\infty}\left|g_{n+1}-g_{n}\right|<\infty, \sum_{n=1}^{\infty}\left|h_{n+1}-h_{n}\right|<\infty$.

Then $\left\{p_{n}\right\},\left\{\phi_{n}\right\}$, and $\left\{\psi_{n}\right\}$ converge strongly to $p_{0}=P_{\mathcal{F}} u$ and $\left(p_{0}, \xi_{0}\right)$ is a solution of (1.6) where $\xi_{0}=P_{C}\left(I-\lambda_{B} D_{2}\right) p_{0}$. 
Proof By using Theorem 3.1 and Lemma 4.6, we obtain the conclusion.

The following result is directly proven from Theorem 4.8. Therefore, we omit the proof.

Corollary 4.9 Let $C$ be a nonempty closed convex subset of a real Hilbert space $H$, let $\Psi_{1}, \Psi_{2}: C \times C \rightarrow \mathbb{R}$ be bifunctions satisfying (J1)-(J4). Let $W$ be a nonspreading mappings of $C$ into itself with $F(W) \neq \emptyset$. Let $D_{1}, D_{2}: C \rightarrow H$ be $d_{1}, d_{2}$-inverse strongly monotone mappings, respectively. Define the mapping $G: C \rightarrow C$ by $G(p)=P_{C}\left(I-\lambda_{A} D_{1}\right)\left(a p+(1-a) P_{C}(I-\right.$ $\left.\lambda_{B} D_{2}\right) p$ ) for all $p \in C$ and $a \in[0,1]$. Assume $\mathcal{F}=\mathrm{EP}\left(\Psi_{1}\right) \cap \operatorname{EP}\left(\Psi_{2}\right) \cap F(G) \cap F(W) \neq \emptyset$. Suppose that $p_{1}, u \in C$ and let $\left\{p_{n}\right\},\left\{\phi_{n}\right\}$, and $\left\{\psi_{n}\right\}$ be sequences generated by

$$
\left\{\begin{array}{l}
\Psi_{1}\left(\phi_{n}, \zeta\right)+\frac{1}{g_{n}}\left\langle\zeta-\phi_{n}, \phi_{n}-p_{n}\right\rangle \geq 0, \quad \forall \zeta \in C, \\
\Psi_{2}\left(\psi_{n}, \zeta\right)+\frac{1}{h_{n}}\left\langle\zeta-\psi_{n}, \psi_{n}-p_{n}\right\rangle \geq 0, \quad \forall \zeta \in C, \\
p_{n+1}=\alpha_{n} u+\beta_{n} p_{n}+\gamma_{n} P_{C}\left(I-\lambda_{n}(I-W)\right) \phi_{n}+\delta_{n} G\left(\psi_{n}\right), \quad \forall n \in \mathbb{N},
\end{array}\right.
$$

where the sequences $\lambda_{A} \in\left(0,2 d_{1}\right), \lambda_{B} \in\left(0,2 d_{2}\right)$ and $\left\{\alpha_{n}\right\},\left\{\beta_{n}\right\},\left\{\gamma_{n}\right\},\left\{\delta_{n}\right\} \subseteq[0,1]$ with $\alpha_{n}+$ $\beta_{n}+\gamma_{n}+\delta_{n}=1$ for all $n \in \mathbb{N}$. Suppose the following conditions hold:

(i) $\lim _{n \rightarrow \infty} \alpha_{n}=0$ and $\sum_{n=1}^{\infty} \alpha_{n}=\infty$,

(ii) $0<c \leq \beta_{n}, \gamma_{n}, \delta_{n} \leq d<1$ for some $c, d>0$ and for all $n \geq 1$,

(iii) $0<e \leq g_{n}, h_{n} \leq$ for some $e, f>0$ and for all $n \geq 1$,

(iv) $\sum_{n=1}^{\infty} \lambda_{n}<\infty$ and $0<\lambda_{n}<1$,

(v) $\sum_{n=1}^{\infty}\left|\alpha_{n+1}-\alpha_{n}\right|<\infty, \sum_{n=1}^{\infty}\left|\beta_{n+1}-\beta_{n}\right|<\infty, \sum_{n=1}^{\infty}\left|\gamma_{n+1}-\gamma_{n}\right|<\infty$, $\sum_{n=1}^{\infty}\left|\lambda_{n+1}-\lambda_{n}\right|<\infty, \sum_{n=1}^{\infty}\left|g_{n+1}-g_{n}\right|<\infty, \sum_{n=1}^{\infty}\left|h_{n+1}-h_{n}\right|<\infty$.

Then $\left\{p_{n}\right\},\left\{\phi_{n}\right\}$, and $\left\{\psi_{n}\right\}$ converge strongly to $p_{0}=P_{\mathcal{F}} u$ and $\left(p_{0}, \xi_{0}\right)$ is a solution of (1.6) where $\xi_{0}=P_{C}\left(I-\lambda_{B} D_{2}\right) p_{0}$.

\section{Example and numerical results}

In this section, we give an example supporting Theorem 3.1.

Example 5.1 Let $\mathbb{R}$ be the set of real numbers and let the mapping $D_{1}, D_{2}: \mathbb{R} \rightarrow \mathbb{R}$ defined by $D_{1} p=\frac{p-2}{3}$ and $D_{2} p=\frac{p-2}{5}, \forall p \in \mathbb{R}$, respectively. Let the mapping $W: \mathbb{R} \rightarrow \mathbb{R}$ be defined by $W p=\frac{p+2}{2}, \forall p \in \mathbb{R}$, let $\Psi_{1}, \Psi_{2}: \mathbb{R} \times \mathbb{R} \rightarrow \mathbb{R}$ be defined by

$$
\Psi_{1}(p, \zeta)=-(p-\zeta)(-4+p+\zeta), \quad \forall p, \zeta \in \mathbb{R}
$$

and

$$
\Psi_{2}(p, \zeta)=-2(p-2)^{2}+(p-2)(\zeta-2)+(\zeta-2)^{2}, \quad \forall p, \zeta \in \mathbb{R}
$$

By the definition of $\Psi_{1}$, we have

$$
\begin{aligned}
0 & \leq \Psi_{1}\left(\phi_{n}, \zeta\right)+\frac{1}{g_{n}}\left\langle\zeta-\phi_{n}, \phi_{n}-p_{n}\right\rangle \\
& =-\left(\phi_{n}-\zeta\right)\left(-4+\phi_{n}+\zeta\right)+\frac{1}{g_{n}}\left(\zeta-\phi_{n}\right)\left(\phi_{n}-p_{n}\right) \\
& =-\left(\phi_{n}-\zeta\right)\left(-4+\phi_{n}+\zeta\right)+\frac{1}{g_{n}}\left(\zeta \phi_{n}-\zeta p_{n}-\phi_{n}^{2}+\phi_{n} p_{n}\right)
\end{aligned}
$$




$$
\begin{aligned}
\Leftrightarrow \quad 0 & \leq-g_{n}\left(\phi_{n}-\zeta\right)\left(-4+\phi_{n}+\zeta\right)+\left(\zeta \phi_{n}-\zeta p_{n}-\phi_{n}^{2}+\phi_{n} p_{n}\right) \\
& =4 g_{n} \phi_{n}-\phi_{n}^{2}-g_{n} \phi_{n}^{2}+\phi_{n} p_{n}+\left(-4 g_{n}+\phi_{n}-p_{n}\right) \zeta+g_{n} \zeta^{2} .
\end{aligned}
$$

Let $G(\zeta)=g_{n} \zeta^{2}+\left(-4 g_{n}+\phi_{n}-p_{n}\right) \zeta+4 g_{n} \phi_{n}-\phi_{n}^{2}-g_{n} \phi_{n}^{2}+\phi_{n} p_{n}$, which is a quadratic function of $\zeta$ with coefficient $a=g_{n}, b=-4 g_{n}+\phi_{n}-p_{n}$, and $c=4 g_{n} \phi_{n}-\phi_{n}^{2}-g_{n} \phi_{n}^{2}+\phi_{n} p_{n}$. Determine the discriminant $\Delta$ of $G$ as follows:

$$
\begin{aligned}
\Delta & =b^{2}-4 a c \\
& =\left(-4 g_{n}+\phi_{n}-p_{n}\right)^{2}-4 g_{n}\left(4 g_{n} \phi_{n}-\phi_{n}^{2}-g_{n} \phi_{n}^{2}+\phi_{n} p_{n}\right) \\
& =16 g_{n}^{2}-8 g_{n} \phi_{n}-16 g_{n}^{2} \phi_{n}+\phi_{n}^{2}+4 g_{n} \phi_{n}^{2}+4 g_{n}^{2} \phi_{n}^{2}+8 g_{n} p_{n}-2 \phi_{n} p_{n}-4 g_{n} \phi_{n} p_{n}+p_{n}^{2} \\
& =\left(-4 g_{n}+\phi_{n}+2 g_{n} \phi_{n}-p_{n}\right)^{2} .
\end{aligned}
$$

We know that $G(\zeta) \geq 0, \forall \zeta \in \mathbb{R}$. If it has at most one solution in $\mathbb{R}$, then $\Delta \leq 0$. So we obtain

$$
\phi_{n}=\frac{4 g_{n}+p_{n}}{1+2 g_{n}} .
$$

By using the same method as (5.1), we have

$$
\psi_{n}=\frac{6 h_{n}+p_{n}}{1+3 h_{n}}
$$

Let $p_{1}, u \in \mathbb{R}$, and $\left\{p_{n}\right\}$ be generated by (3.1) as follows:

$$
\left\{\begin{array}{l}
\Psi_{1}\left(\phi_{n}, \zeta\right)+\frac{1}{g_{n}}\left\langle\zeta-\phi_{n}, \phi_{n}-p_{n}\right\rangle \geq 0, \quad \forall \zeta \in C, \\
\Psi_{2}\left(\psi_{n}, \zeta\right)+\frac{1}{h_{n}}\left\langle\zeta-\psi_{n}, \psi_{n}-p_{n}\right\rangle \geq 0, \quad \forall \zeta \in C, \\
p_{n+1}=\alpha_{n} u+\beta_{n} p_{n}+\gamma_{n} P_{C}\left(I-\lambda_{n}(I-W)\right) \phi_{n}+\delta_{n} G\left(\psi_{n}\right), \quad \forall n \in \mathbb{N},
\end{array}\right.
$$

where $a=0.5, \lambda_{A}=1, \lambda_{B}=1, g_{n}=\frac{n}{3 n+1}, h_{n}=\frac{n}{4 n+1}, \alpha_{n}=\frac{1}{2 n}, \beta_{n}=\frac{3 n-1}{16 n}, \gamma_{n}=\frac{10 n-3}{16 n}, \delta_{n}=\frac{3 n-4}{16 n}$, and $\lambda_{n}=\frac{1}{2 n^{2}}$ for all $n \in \mathbb{N}$. By the definitions of $\Psi_{1}, \Psi_{2}, G$, and $W$, we have $\operatorname{EP}\left(\Psi_{1}\right) \cap$ $\mathrm{EP}\left(\Psi_{2}\right) \cap F(G) \cap F(W)=\{2\}$. From Theorem 3.1, we can conclude that the sequences $\left\{p_{n}\right\}$, $\left\{\phi_{n}\right\}$, and $\left\{\psi_{n}\right\}$ converge strongly to 2 . From (5.1) and (5.2), we can rewrite (3.1) as follows:

$$
\left\{\begin{array}{l}
\phi_{n}=\frac{4 g_{n}+p_{n}}{1+2 g_{n}}, \\
\psi_{n}=\frac{6 h_{n}+p_{n}}{1+3 h_{n}}, \\
p_{n+1}=\frac{1}{2 n} u+\frac{3 n-1}{16 n} p_{n}+\frac{10 n-3}{16 n}\left(I-\frac{1}{2 n^{2}}(I-W)\right) \phi_{n}+\frac{3 n-4}{16 n} G\left(\psi_{n}\right), \quad \forall n \geq 1 .
\end{array}\right.
$$

Table 1 shows the values of the sequences $\left\{p_{n}\right\},\left\{\phi_{n}\right\}$, and $\left\{\psi_{n}\right\}$ where $u=p_{1}=-1$ and $u=p_{1}=5$ and $n=300$.

\section{Conclusion}

1. The sequences $\left\{p_{n}\right\},\left\{\phi_{n}\right\}$, and $\left\{\psi_{n}\right\}$ in Table 1 and Figure 1 converge to 2 , where $\{2\}=\mathrm{EP}\left(\Psi_{1}\right) \cap \mathrm{EP}\left(\Psi_{2}\right) \cap F(G) \cap F(W)$.

2. Theorem 3.1 ensures the convergence of $\left\{p_{n}\right\},\left\{\phi_{n}\right\}$, and $\left\{\psi_{n}\right\}$ in Example 5.1. 
Table 1 The values of $\left\{\phi_{n}\right\},\left\{\psi_{n}\right\}$, and $\left\{p_{n}\right\}$ where $n=300$

\begin{tabular}{|c|c|c|c|c|c|c|}
\hline \multirow[t]{2}{*}{$n$} & \multicolumn{3}{|c|}{$u=p_{1}=-1$} & \multicolumn{3}{|l|}{$u=p_{1}=5$} \\
\hline & $\phi_{n}$ & $\psi_{n}$ & $p_{n}$ & $\phi_{n}$ & $\psi_{n}$ & $p_{n}$ \\
\hline 1 & 0.0000 & 0.1250 & -1.0000 & 4.000000 & 3.8750 & 5.0000 \\
\hline 2 & 0.4339 & 0.5234 & -0.4609 & 3.5661 & 3.4766 & 4.4609 \\
\hline 3 & 0.7688 & 0.8360 & 0.0301 & 3.2312 & 3.1640 & 3.9699 \\
\hline 4 & 1.0254 & 1.0771 & 0.4256 & 2.9746 & 3.9229 & 3.5744 \\
\hline 5 & 1.2188 & 1.2595 & 0.7306 & 2.7812 & 2.7405 & 3.2694 \\
\hline & : & & & & & \\
\hline 150 & 1.9837 & 1.9845 & 1.9728 & 2.0163 & 2.0155 & 2.0272 \\
\hline$\vdots$ & $\vdots$ & $:$ & & & $\vdots$ & \\
\hline 296 & 1.9918 & 1.9922 & 1.9863 & 2.0082 & 2.0078 & 2.0137 \\
\hline 297 & 1.9918 & 1.9922 & 1.9864 & 2.0082 & 2.0078 & 2.0136 \\
\hline 298 & 1.9918 & 1.9922 & 1.9864 & 2.0082 & 2.0078 & 2.0136 \\
\hline 299 & 1.9919 & 1.9923 & 1.9865 & 2.0081 & 2.0077 & 2.0135 \\
\hline 300 & 1.9919 & 1.9923 & 1.9865 & 2.0081 & 2.0077 & 2.0135 \\
\hline
\end{tabular}

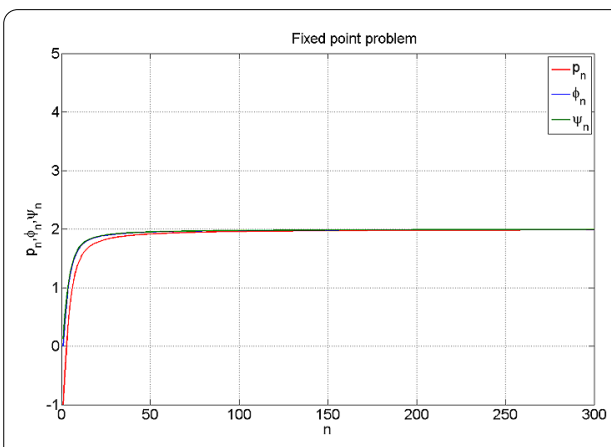

(a) $u=p_{1}=-1$

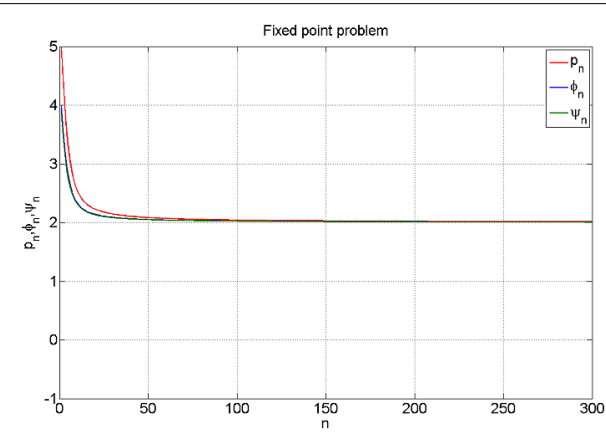

(b) $u=p_{1}=5$

Figure 1 The convergence comparison of the sequences $\left\{p_{n}\right\},\left\{\phi_{n}\right\}$, and $\left\{\psi_{n}\right\}$ with different initial values $u$ and $p_{1}$.

\section{Competing interests}

The authors declare that they have no competing interests.

\section{Authors' contributions}

The authors contributed equally and significantly in writing this paper. All authors read and approved the final manuscript.

\section{Author details}

'Department of Mathematics, Faculty of Science, King Mongkut's Institute of Technology Ladkrabang, Bangkok 10520,

Thailand. ${ }^{2}$ Department of Mathematics, Faculty of Science, Chiang Mai University, Chiang Mai, 50200, Thailand.

\section{Acknowledgements}

This paper was supported by the Thailand Research Fund under the research project RTA578007 and the Research Administration Division of King Mongkut's Institute of Technology Ladkrabang.

\section{Received: 7 April 2015 Accepted: 3 November 2015 Published online: 25 November 2015}

\section{References}

1. Blum, E, Oettli, W: From optimization and variational inequalities to equilibrium problems. Math. Stud. 63(14), 123-145 (1994)

2. Lions, JL, Stampacchia, G: Variational inequalities. Commun. Pure Appl. Math. 20, $493-517$ (1967)

3. Diaz, JB, Metcalf, FT: On the structure of the set of subsequential limit points of successive approximations. Bull. Am. Math. Soc. 73, 516-519 (1967)

4. Su, Y, Wang, D, Shang, M: Strong convergence of monotone hybrid algorithm for quasi-nonexpansive mappings. Int. J. Math. Anal. 1, 1235-1241 (2007)

5. Tian, M, Jin, X: Strong convergent result for quasi-nonexpansive mappings in Hilbert spaces. Fixed Point Theory Appl. 2011, 88 (2011) 
6. Dong, QL, Guo, YN, Su, F: Hybrid iterative scheme by a relaxed extragradient method for equilibrium problems, a general system of variational inequalities and fixed point problems of a countable family of nonexpansive mappings. J. Appl. Math. 2012, Article ID 572326 (2012)

7. Ceng, LC, Wang, CY, Yao, JC: Strong convergence theorems by a relaxed extragradient method for a general system of variational inequalities. Math. Methods Oper. Res. 67, 375-390 (2008)

8. Kangtunyakarn, A: An iterative algorithm to approximate a common element of the set of common fixed points for a finite family of strict pseudo-contractions and of the set of solutions for a modified system of variational inequalities. Fixed Point Theory Appl. 2013, 143 (2013)

9. Opial, Z: Weak convergence of the sequence of successive approximation of nonexpansive mappings. Bull. Am. Math. Soc. 73, 591-597 (1967)

10. Osilike, MO, Igbokwe, DI: Weak and strong convergence theorems for fixed points of pseudocontractions and solutions of monotone type operator equations. Comput. Math. Appl. 40, 559-567 (2000)

11. Combettes, PL, Hirstoaga, SA: Equilibrium programming in Hilbert spaces. J. Nonlinear Convex Anal. 6(1), 117-136 (2005)

12. Xu, HK: An iterative approach to quadric optimization. J. Optim. Theory Appl. 116, 659-678 (2003)

13. Takahashi, W: Nonlinear Functional Analysis. Yokohama Publishers, Yokohama (2000)

14. Cheawchan, $K$, Kangtunyakarn, A: Approximation method for fixed points of nonlinear mapping and variational inequalities with application. Thai J. Math. (in press)

15. Takahashi, S, Takahashi, T: Viscosity approximation methods for equilibrium problems and fixed point problems in Hilbert spaces. J. Math. Anal. Appl. 331, 506-515 (2007)

16. Kohsaka, F, Takahashi, W: Fixed point theorems for a class of nonlinear mappings related to maximal monotone operators in Banach spaces. Arch. Math. 91, 166-177 (2008)

17. lemoto, S, Takahashi, W: Approximating common fixed points of nonexpansive mappings and nonspreading mappings in a Hilbert space. Nonlinear Anal. 71, 2082-2089 (2009)

18. Kangtunyakarn, A, Suantai, S: Hybrid iterative scheme for generalized equilibrium problems and fixed point problems of finite family of nonexpansive mappings. Nonlinear Anal. Hybrid Syst. 3, 296-309 (2009)

19. Kangtunyakarn, A, Suantai, S: A new mapping for finding common solutions of equilibrium problems and fixed point problems of finite family of nonexpansive mappings. Nonlinear Anal. 71, 4448-4460 (2009)

20. Kangtunyakarn, A: Strong convergence of the hybrid method for a finite family of nonspreading mappings and variational inequality problem. Fixed Point Theory Appl. 2012, 188 (2012)

\section{Submit your manuscript to a SpringerOpen ${ }^{\circ}$ journal and benefit from:}

- Convenient online submission

Rigorous peer review

- Immediate publication on acceptance

- Open access: articles freely available online

- High visibility within the field

- Retaining the copyright to your article 